\title{
Les perspectives de résidents concernant les soins aux personnes en situation de pauvreté. Utilisation du photovoix comme dispositif réflexif d'analyse de besoins de formation
}

\section{Resident outlook on providing care to people living in poverty. The use of the photovoice reflexive analysis for training purposes}

\author{
Annie-Claude MAILHOT ${ }^{1}$, Christine LOIGNON ${ }^{2}$, Caroline MASSICOTTE $^{3}$, \\ Rébecca MILLETTE ${ }^{4}$, Julie SIROIS-LECLERC ${ }^{5}$, Yanouchka LABROUSSE $^{6}$, \\ Bruno FORTIN ${ }^{6}$, Karoline TRUCHON ${ }^{7}$ et Alexandrine BOUDREAULT-FOURNIER ${ }^{8}$ \\ 1 Centre local de services communautaires (CLSC) Samuel de Champlain, Brossard, Canada \\ Département de médecine de famille et de médecine d'urgence, Université de Sherbrooke, Longueuil, Canada \\ Clinique de santé Jacques-Cartier, Sherbrooke, Canada \\ GMF Ahunstic-Clinique Pas-à-Pas, Montréal-Nord, Canada \\ Médecin de famille en pratique active sans affiliation au Québec, Canada \\ 6 Unité de médecine familiale Charles-Lemoyne, Saint-Lambert, Canada \\ 7 Département d'anthropologie, Université Laval, Québec Canada \\ 8 Département d'anthropologie, University of Victoria, Victoria, Canada
}

Manuscrit soumis le 21 janvier 2014 ; commentaires éditoriaux formulés aux auteurs le 14 mars 2016 ; accepté pour publication le 2 juillet 2016

\author{
Mots-clés \\ Méthode réflexive \\ d'apprentissage ; \\ photovoix ; \\ perspectives de \\ résidents ; pauvreté
}

Résumé - Contexte et problématique : Les perceptions, croyances et attitudes des médecins envers les personnes en situation de pauvreté constituent des éléments ayant des impacts significatifs sur la qualité des soins. Or, au Québec, il existe peu de formation outillant les médecins de famille à soigner les personnes vivant en situation de pauvreté. Objectif : Cette étude visait à explorer les besoins en termes de formations des résidents concernant les soins auprès des personnes en situation de pauvreté. Matériel et méthode : Cette étude a utilisé un devis de recherche qualitatif de type recherche-action avec la méthode photovoix. Quatre résidentes en médecine familiale ont été accompagnées pendant le processus par une sociologue superviseure, une anthropologue spécialiste du visuel, un psychologue et un médecin. Résultats : Le 
Keywords

Reflexive learning method; photovoice; resident outlook; poverty processus de photovoix a fait ressortir trois barrières interférant dans la relation entre le résident et la personne en situation de pauvreté : 1) le contexte de la pratique médicale du résident ; 2) le contexte socio-économique du patient ; et 3) la distance sociale entre les médecins et les personnes en situation de pauvreté. Discussion : Le photovoix a généré une pédagogie de l'inconfort, manière d'être qui augmente la possibilité de connaissance des conditions réelles des personnes en situation de pauvreté par l'empathie des résidentes-sujetschercheures. Conclusion : Cette étude a permis d'identifier trois principales barrières qui interviennent entre les résidents et les personnes en situation de pauvreté. Elle a également permis l'identification de solutions qui, selon les résidentes, permettraient de les dépasser.

Abstract - Background and issues: Physicians' perceptions, beliefs and attitudes towards people living in poverty seriously impact the quality of care. In Quebec, there is little training to help family physicians care for people living in poverty. Purpose: This study aims to examine the training needed by medical residents when providing care to people in poverty. Material and method: The study drew on a qualitative research approach, more specifically a research-action using the photovoice method. During the process, a supervising sociologist, a visual anthropologist, a psychologist and a physician oversaw four residents in family medicine. Results: The photovoice process highlighted three challenges playing a role in the relation between residents and people living in poverty: 1) the context of the resident's medical practice; 2) the patient's socio-economic context; and 3) the social distance between the physicians and the people in poverty. Discussion: The photovoice produced a pedagogy of discomfort, i.e. a way of being that could potentially broaden knowledge of the real conditions of people in poverty as a result of the empathy shown by residents/subjects/researchers. Conclusion: The study helped ascertain the three main challenges that influence the relationship between the residents and people living in poverty. According to the residents it further helped identify solutions that were instrumental in resolving issues.

\section{Introduction}

La pauvreté au Canada, de même qu' ailleurs dans le monde, est un déterminant de la santé incontournable ${ }^{[1,2]}$. Il est fondamental que les médecins de famille comprennent ses effets sur la santé et puissent trouver des solutions pour en limiter les impacts négatifs. Dans ce contexte, les perceptions, croyances et attitudes des médecins envers les personnes en situation de pauvreté ont été identifiées comme étant des éléments ayant un impact déterminant sur la qualité des soins. Comme les médecins ont généralement une image négative de l'attitude des personnes en situation de pauvreté envers la santé, ils agissent souvent de manière paternaliste ${ }^{[3]}$, donnent moins d'informations et consacrent moins de temps à celles-ci ${ }^{[4]}$.

Au Québec, de même qu'au Canada, il existe très peu de cours ou de stages de formation visant à outiller les médecins de famille à soigner les personnes vivant en situation de pauvreté. Des méthodes pédagogiques porteuses ont été identifiées pour mieux former les médecins à soigner les personnes en situation de pauvreté et éviter les attitudes négatives à leur égard. Parmi celles-ci, notons l'inclusion des connaissances sur la pauvreté et les inégalités sociales dans le curriculum des médecins en formation $^{[5]}$ et l'intégration des méthodes d'apprentissage actives (activités réflexives, contacts répétés avec la communauté, etc.). On estime que ces méthodes pourraient avoir un impact positif sur les attitudes des médecins envers les personnes en situation de pauvreté et donc contribuer à l'amélioration de la qualité des soins ${ }^{[3]}$.

\section{Contexte et problématique}

Dans cette étude, il est entendu par personne en situation de pauvreté tout individu qui bénéficie de prestations d'aide sociale ou qui dispose d'un revenu qui se situe en deçà du seuil de faible revenu selon l'indice LICO (low income cut-off) de Statistiques 
Canada. Au Canada, en 2006, 4.6 millions de personnes vivaient en situation de pauvreté, soit $14,5 \%$ de la population ${ }^{[8]}$. Les personnes en situation de pauvreté sont plus à risque de développer des maladies d'ordre physique et psychologique ${ }^{[6]}$, et de présenter de multiples comorbidités ${ }^{[4]}$. Elles ont également un plus grand risque de chronicisation de leurs problèmes de santé ${ }^{[6]}$. En fait, $40 \%$ des Canadiens ayant un faible revenu souffrent d'une maladie chronique $^{[7]}$. Cependant, les personnes en situation de pauvreté sont parmi les moins bien desservies sur le plan des soins primaires préventifs, au Canada de même qu'ailleurs dans le monde. Dans la majorité des cas, elles mentionnent avoir l'impression que leurs besoins ne sont pas comblés ${ }^{[6]}$. Par ailleurs, ces patients se sentent souvent intimidés, dominés ou stigmatisés par leur médecin ${ }^{[7]}$. Il semblerait que les patients qui se sentent dévalorisés dans la relation avec leur médecin se perçoivent encore plus démunis devant les difficultés associées à la pauvretét ${ }^{[2]}$. En somme, l'obtention de soins de santé adéquats est plus difficile pour les personnes en situation de pauvreté.

En 2011, à la Faculté de médecine de l'Université de Sherbrooke, une recherche-action s'inscrivant dans le sillage des travaux de Loignon et al. ${ }^{[6,7,8]}$ sur le développement de la compétence sociale a été entamée ${ }^{[9,10]}$.

Objectif

L'objectif de cette recherche-action visait à explorer les besoins en termes de formation des résidents concernant les soins auprès des personnes en situation de pauvreté. La question de recherche déterminée par les résidents en collaboration avec les superviseurs et les chercheurs était la suivante : quelles sont les barrières qui subsistent entre les médecins en formation et les personnes en situation de pauvreté?

\section{Matériel et méthodes}

Les participantes

Ce projet comprenait huit participantes. L'équipe de recherche était constituée de quatre résidentes en médecine familiale de l'Hôpital Charles-LeMoyne pratiquant en clinique et en hôpital auprès de patients provenant de milieux socio-économiques diversifiés. Les résidentes ont été sélectionnées sur une base volontaire, sans critère d'inclusion ou d'exclusion et sans égard à l'expérience personnelle actuelle ou passée de contact avec la pauvreté. Chacune d'entre elles agissait à la fois comme sujet et chercheure au cours de l'étude (Annie-Claude Maillhot, Caroline Massicotte, Rébecca Millette, Julie Sirois-Leclerc). Les résidentes-sujets-chercheures ont été accompagnées pendant le processus par une sociologue (Christine Loignon), qui supervisait également le projet, et par une anthropologue spécialiste du visuel (Alexandrine Boudreault-Fournier), en présence d'un psychologue (Bruno Fortin) et d'un médecin (Yanouchka Labrousse), pratiquant tous deux à l'unité de médecine familiale de l'Hôpital Charles-LeMoyne, situé sur la Rive-Sud de Montréal au Québec. Cette étude a été approuvée par le comité d'éthique du Centre hospitalier Universitaire de Sherbrooke (CHUS) et les huit participants ont signé un formulaire de consentement.

\section{Le photovoix comme méthode de formation et de réflexivité}

Cette étude a utilisé un devis de recherche qualitatif de type recherche-action participative axée sur le transfert des connaissances. Elle a mobilisé une approche méthodologique, la méthode "photovoice », ici traduite et appelée " photovoix ». Le photovoix est défini comme un procédé par lequel les personnes peuvent s'identifier et représenter les membres de leur communauté à travers une technique photographique ${ }^{[11]}$. Concrètement, il s'agit de donner une caméra à une certaine population, l'inviter à prendre des photographies de son vécu et, finalement, se réunir pour discuter des photographies ${ }^{[11,12]}$.

Le procédé a trois objectifs : permettre à la population d'exprimer le point de vue de leur communauté, promouvoir un dialogue critique et échanger des connaissances et, idéalement, rejoindre ensuite des autorités décisionnelles (souvent par le biais d'une exposition des photographies produites par les 
participants) ${ }^{[11]}$. Ainsi, la méthode photovoix s'inscrit dans la lignée des différentes méthodes participatives qui ont pour but de favoriser la responsabilisation (empowerment) des membres d'une communauté envers eux-mêmes et les problèmes vécus au sein de leur communauté.

Bien que les manières de réaliser les photovoix varient d'une étude à l'autre, chaque projet tente de répondre à une question de nature descriptive afin de pouvoir proposer des solutions de nature prescriptive. La principale source d'analyse n'est généralement pas les photographies, mais plutôt la discussion entre les participants au sujet de celles-ci ${ }^{[11]}$. La prise de photographies est donc un déclencheur de réflexion et la discussion qui la suit, un révélateur des divers aspects articulant les réflexions individuelles et de groupe des participants impliqués. En conséquence, plusieurs auteurs estiment qu'il est nécessaire d'impliquer les participants dans l'analyse et l'interprétation des données afin de refléter correctement leurs opinions ${ }^{[12]}$. Les principaux impacts de cette méthode de recherche sont une meilleure compréhension des besoins du milieu, la responsabilisation des participants (empowerment) et l'atteinte des autorités décisionnelles ${ }^{[11]}$

Dans le cadre de cette étude, la méthode photovoix appliquée dans le contexte des barrières entre les médecins en formation et les personnes en situation de pauvreté a permis de mieux comprendre la complexité des perceptions des résidentes-sujetschercheurs à l'égard de ces personnes.

La collecte des données avec les rencontres de photovoix

Six rencontres ont été organisées de janvier à mai 2011 entre les résidentes-sujets-chercheurs (RSC) et les superviseures cliniques, une sociologue et une anthropologue du visuel. La première rencontre a permis de faire ressortir et de préciser la question de recherche et de mieux comprendre la méthode photovoix. Par la suite, les résidentessujets-chercheures ont eu trois semaines pour procéder à la collecte de données qui consistait à prendre chacune environ dix à quinze photos représentant la conception qu'elles se faisaient des barrières qui existent entre les médecins et les personnes en situation de pauvreté. Chacune des résidentes-sujetschercheures a ensuite sélectionné un maximum de cinq photos qui représentaient le mieux leur conception personnelle de ces barrières.

Afin de faire émerger les données, un groupe de discussion a été formé pour une durée de deux heures. Pendant cette séance, les vingt photos sélectionnées ont été présentées par les résidentes-sujets-chercheures, puis commentées en groupe. La rencontre était animée par la sociologue et l'anthropologue spécialiste du visuel, en présence du psychologue et du médecin. Chacune des personnes présentes a participé activement à la discussion, amenant ainsi des points de vue teintés d'une expérience, d'une sensibilisation à la pauvreté et d'un passé différents. Le contenu de cette rencontre a été enregistré à l'aide d'un appareil audio numérique et retranscrit intégralement sous forme de verbatim pour les besoins de l'analyse.

\section{Rencontrer et dialoguer avec des personnes en situation de pauvreté}

En parallèle au projet photovoix, lors de la période de l'analyse des données, deux des RSC ont participé à une soirée à l'Université populaire d'ATD Quart-Monde à Montréal. L'objectif d'ATD Quart-Monde est de permettre l'accès aux personnes les plus pauvres au plein exercice de leurs droits et de favoriser des stratégies menant à l'éradication de pauvreté. L’Université Quart-Monde offre donc aux personnes en situation de pauvreté de se rassembler pour exprimer et réfléchir sur la pauvreté et sur les dimensions structurelles et sociétales qui la leur impose. Elle offre également des possibilités d'échange avec des personnes ne vivant pas une situation de pauvreté. Dans cette rencontre, dont le thème était «Le bonheur », les RSC ainsi que la sociologue superviseure ont eu l'occasion d'échanger avec des personnes en situation de pauvreté sur leur vision du bonheur individuel et collectif. Cette activité a permis à ces participantes de développer une sensibilisation accrue face à la problématique de 
la pauvreté, sensibilité qui a été mise à contribution pendant l'analyse des données et la rédaction du rapport d'érudition.

\section{L'analyse des données}

L'analyse de contenu de type conventionnel a été utilisée et développée. Ce type d'analyse permet d'acquérir des informations directement des participants sans imposer des catégories préconçues ou des perspectives théoriques ${ }^{[13]}$. La superviseure sociologue et l'anthropologue spécialiste du visuel ont formé les résidentes-sujets-chercheures à l'analyse de données qualitatives et ont collaboré à toutes les étapes de l'analyse. Les RSC ont procédé à l'organisation des données via un processus de codification. L'analyse des données consistait à effectuer plusieurs lectures de la transcription de la rencontre où les RSC présentaient et échangeaient sur les photos. Une rencontre a été ensuite consacrée à l'interprétation des données afin de faire émerger les principales barrières entre les personnes en situation de pauvreté et les médecins en formation avec les discussions suscitées par les différentes photos. À partir des codes identifiés de manière inductive, le contenu de la transcription a ensuite été codifié et analysé par les quatre RSC, d'abord individuellement, puis en équipe de deux, et enfin en groupe. Une grille d'analyse du texte a été élaborée en faisant ressortir les citations les plus représentatives des barrières préalablement identifiées. Quelques pistes de solutions ont également découlé de l'analyse des données de cette rencontre, comme thème émergent, bien que l'objectif de recherche ne fût pas centré sur cet aspect.

À la suite de cette première organisation du contenu de la transcription, la grille d'analyse a été développée par la sociologue superviseure et l'anthropologue spécialiste du visuel. Elles ont proposé trois principales catégories de barrières qui ont par la suite été discutées et approuvées par les RSC. Ces dernières ont ensuite rédigé les synthèses de résultats.

La crédibilité des résultats a été assurée par une triangulation des données par la codification intercodeur et la multiplication des sources de données, par exemple, les transcriptions des discussions en groupe et la rédaction de notes par les chercheurs, de même que leur implication lors de l'analyse des données. Le fait que les RSC aient été formées et impliquées lors de l'analyse des données et soutenues par les accompagnateurs (superviseure sociologue, anthropologue spécialiste du visuel, psychologue et médecin) lors de plusieurs rencontres a permis de rehausser la qualité de la réflexivité et le respect des divergences au niveau de l'analyse tout en confirmant la perspective des participants.

\section{Résultats}

Le processus de photovoix a permis de faire ressortir trois types de barrières pouvant interférer dans la relation entre le médecin en formation et la personne en situation de pauvreté : 1) le contexte de la pratique médicale du médecin en formation ; 2) le contexte socio-économique du patient et : 3 ) la distance sociale entre les médecins qui dispensent les soins et les personnes en situation de pauvreté.

\section{Barrière 1: le contexte de la pratique médicale des résidents}

Premièrement, plusieurs éléments étaient relatifs au contexte de pratique des médecins en formation. Une barrière importante identifiée lors du photovoix fut le manque de formation des étudiants et résidents en médecine en rapport avec les personnes en situation de pauvreté. Plus précisément, un élément manquant dans la formation médicale est la connaissance des ressources dont les personnes en situation de pauvreté pourraient grandement bénéficier. À ce sujet, la RSC2 a affirmé : "Je ne connais [...] vraiment pas les ressources pour les personnes dans ces situations-là, [...] c'est quelque chose qu'il faudrait que j'apprenne à savoir, où aller rechercher [...] je connais pas vraiment les endroits où référer ».

D'un point de vue plus organisationnel, les RSC ont identifié le manque de temps comme facteur important pouvant entraver la relation thérapeutique avec la personne en situation de pauvreté. RSC3 a 


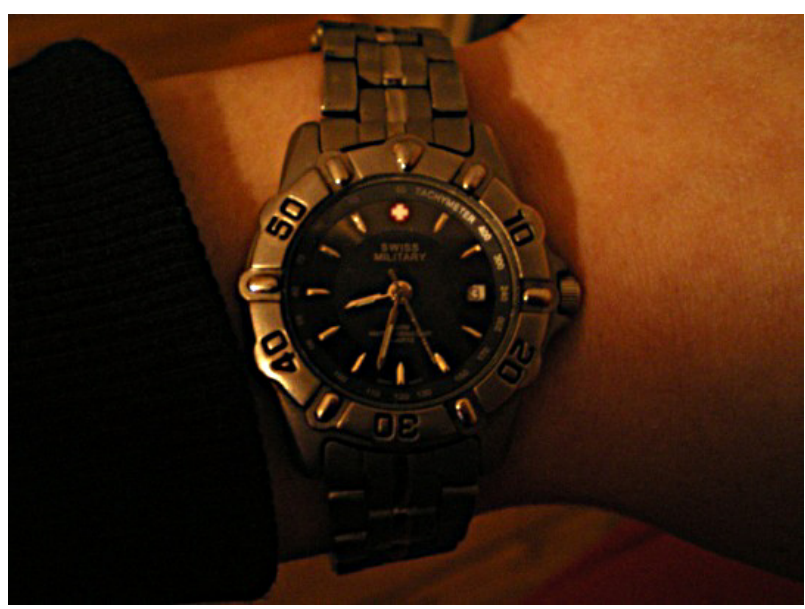

Photo 1. Le manque de temps comme barrière.

souligné : «On essaie de gérer notre temps avec nos patients, mais encore plus avec ces patients-là parce que souvent l'entrevue va être un peu plus longue parce qu'ils ont plus de besoins, ou il va falloir donner plus d'information, ou il va falloir se réorienter dans le traitement » (voir Photo 1).

Dans ce contexte, selon les participantes, le résident pourrait se sentir mal outillé lorsqu'il est confronté à tous les besoins de la personne vivant en situation de pauvreté et au court laps de temps accordé pour pouvoir les combler. Cette situation est d'autant plus notable que les participantes ont mentionné que dans certains milieux, le résident n'a aucun pouvoir décisionnel sur la durée des rencontres, et ce même s'il juge important d'accorder un peu plus de temps pour une personne en situation de pauvreté en particulier. RSC4 a stipulé que «pour le résident, c'est le temps [en parlant des barrières] parce que nous, on a un temps imposé ». Cette RSC a ajouté : "souvent, je trouve que les patients dans une situation de pauvreté comme ça, ils ont une anxiété ou une angoisse ou quelque chose de sousjacent qu'on ne découvre pas, puis si on avait une heure, probablement qu'on y arriverait. Puis là, on n'y arrive pas. »

D’une façon plus générale, le sentiment de devoir soigner le plus de patients possible dans un temps donné est présent pour de multiples raisons. L'une de celles-ci est la forte demande faite par la population qui démontre un besoin grandissant d'être suivi par un médecin de famille. Par conséquent, cela limite le temps pouvant être accordé à chacun des patients. RSC2 a exprimé qu'il est difficile de "gérer notre temps, on n'a pas le choix. Il faut toujours qu'on prenne en compte les besoins de la collectivité. Tu sais, on ne peut pas prendre tout le temps pour un patient. »

Dans le même ordre d'idées, un autre facteur qui influe sur le temps consacré à chacun des patients est l'incitatif salarial car la majorité des médecins sont rémunérés par acte accompli au Québec. Ainsi, la majorité des médecins en formation seront soumis aux mêmes règles et donc, pour être avantagé dans sa pratique future, le résident est formé afin d'être en mesure de voir beaucoup de patients sur une plus courte période de temps. Pour RSC3, "dans une clinique où les médecins sont payés à l'acte, comme la plupart des médecins de famille, bien, ça devient un obstacle, la façon dont on rémunère, parce que c'est l'examen physique qu'ils payent; ça fait que tu peux faire tout le blabla, puis la compréhension de son contexte psychosocial...». Elle a renchérit en mentionnant ceci : "il y a aussi quelque chose de social qui fait que le médecin qui va prendre le temps d'expliquer, de laisser le dictionnaire médical pour voir la réaction du patient, puis justement pour s'adapter, bien, c'est aussi ce qui est le moins valorisé dans l'échelle pyramidale de salaire. »

En somme, les résidents vivent plusieurs obstacles d'ordre contextuels liés à leur environnement de pratique et leur formation lorsqu'ils entrent en relation avec les personnes en situation de pauvreté. Par exemple, des RSC ont témoigné que le manque de connaissance des ressources existantes contribue à leur sentiment d'impuissance lors de leur relation d'aide avec les personnes en situation de pauvreté. De plus, le résident travaille souvent dans un milieu où les médecins doivent consacrer un temps limité à chacun des patients. Dans ce contexte, il peut donc être difficile de développer une bonne alliance thérapeutique avec les personnes en situation de pauvreté. 
Barrière 2 : le contexte socio-économique du patient

Le contexte socio-économique du patient a aussi été identifié comme une des barrières majeures qui subsiste entre les médecins en formation et les personnes en situation de pauvreté. En effet, autant les ressources financières restreintes des personnes en situation de pauvreté que leur situation sociale particulière entravent la prise en charge de leur santé.

D'emblée, il apparaît nécessaire de souligner que le caractère involontaire de la situation économique des personnes en situation de pauvreté est un aspect fondamental. Les RSC différenciaient la "simplicité volontaire », où l'individu choisit de vivre en situation de précarité économique, de la pauvreté, qui fait référence à l'absence de choix et à l'absence de moyens pour pouvoir s'en sortir. RSC1 a mentionné que "la pauvreté c'est vraiment ça. C'est l'absence de choix, l'absence de moyens, qui fait que tu es dans une situation [...] Mais quand tu as le choix de pouvoir t'en sortir, c'est différent. »

Premièrement, la précarité économique implique une accessibilité limitée à un milieu de vie sain, à des habitudes de vie équilibrées et au traitement optimal des conditions médicales. Cet aspect est d'autant plus important que les difficultés économiques semblent être omniprésentes dans le contexte de la pratique médicale au Québec. RSC3 l'a mis en évidence en explorant la situation financière de ses patients : "Je demande : 'comment ça va financièrement?', puis il n'y a personne qui m'a répondu 'bien', personne. »

Au premier abord, le milieu de vie déficient de certaines personnes en situation de pauvreté semble avoir un impact significatif sur la santé, autant par l'aspect de la promiscuité que de l'insalubrité. Par exemple, une famille de cinq personnes qui s'entassent dans la même chambre pour la nuit, ou encore un logement infesté de moisissures peuvent avoir des conséquences significatives sur la santé. RSC4 a informé : "Je suis allée [...] dans un logement qui était insalubre. [...] J'étais vraiment surprise de l'état des lieux qui était absolument catastrophique, puis dans les chambres, il y avait des moisissures partout sur les murs, puis un enfant asthmatique couché sur le lit. »

De plus, les limites budgétaires rendent difficiles l'accès à des aliments sains. La publicité met aussi davantage en valeur ce que nous nommons au Québec comme la "malbouffe » plutôt qu'elle ne met l'emphase sur les fruits et les légumes. En outre, le coût des médicaments ainsi que celui de la psychothérapie, constituent des barrières importantes à la prise de charge de la santé des personnes en situation de pauvreté. Ces dernières vont d'ailleurs avoir tendance à s'automédicamenter avec la cigarette ou par l'alcool. Dans les mots de RSC1: "Le fait d'avoir leurs petites cigarettes, peut-être que ça les aide à décompresser, puis ils n'ont pas l'argent, eux, pour avoir du Celexa [citalopram-antidépresseur inhibiteur sélectif de la recapture de la sérotonine] tous les jours. »

Deuxièmement, le contexte social propre aux personnes en situation de pauvreté limite l'accès aux soins de santé optimal de par leur isolement géographique, leur manque de ressources et leur niveau de littératie. Et bien que l'accès restreint à des moyens de transport constitue en soi une barrière à l'obtention de soins de santé, la répartition géographique des services de santé occupe aussi un aspect central dans l'accès aux soins. Par exemple, une restructuration du réseau des centres locaux de services communautaires (CLSC) ne tenant pas compte des 'quartiers socio-économiques' limite l'accès aux services. En effet, les personnes en situation de pauvreté craignent de se rendre dans le CLSC appartenant à une autre classe socio-économique, dû à un sentiment de honte et de malaise. Pour RSC2 : "Le concept géographique, nous on prend ça un peu comme acquis. Moi, je peux me déplacer à Montréal-Nord, je peux aller dans Westmount, je suis capable d'aller sur Internet voir les autobus, etc. Mais je pense que [...] l'idée d'être près de la maison, de la zone, de connaître où ils vont, c'est aussi très important quand on réfère à des ressources. »

Par ailleurs, il ressort qu'un certain niveau de littératie en santé soit nécessaire afin de pouvoir s'y retrouver et de prendre les bonnes décisions. La pauvreté n'équivaut pas d'emblée à un niveau 
de littératie moindre, mais peut en être un facteur prédisposant.

Du point de vue des soins de santé, c'est autant l'accès au professionnel de la santé qu'au traitement qui se trouve limité en raison de la situation sociale des personnes en situation de pauvreté. Le temps d'attente pour avoir accès aux médecins de deuxième ligne est souvent long, et mieux vaut avoir un titre pour faire avancer son dossier. RSC3 a souligné : "Quand j'appelle pour prendre des rendez-vous pour mes patients, [...] [je me rends] compte de ce que ça prend pour se débrouiller dans le système, alors ça doit être très facile d'être en marge. »

Enfin, il est ressorti que la situation de pauvreté semble amener les personnes à prendre des décisions davantage dans l'immédiat que dans une perspective à long terme. En conséquence, il peut être difficile pour celles-ci d'investir dans la prévention primaire de maladies qui se manifesteront à moyen ou long terme, alors qu'elles ont surtout une perspective immédiate de la santé.

Barrière 3 : la distance sociale entre les médecins qui dispensent les soins et les personnes en situation de pauvreté.

La troisième et dernière barrière identifiée lors de l'analyse de données englobait toute la question de la distance sociale entre les médecins qui dispensent les soins et les personnes en situation de pauvreté. Les médecins et personnes en situation de pauvreté sont issus de milieux socio-économiques et socio-culturels différents, ce qui rend la compréhension de part et d'autre plus problématique, comme le verbalisaient deux RSC lors de la discussion : D'emblée, RSC3 a avancé : "J'ai l'impression que c'est deux mondes séparés et que c'est difficile à concilier. » RSC1 a ajouté : "On est médecin, puis tout le monde sait qu'on fait un bon salaire, c'est pas quelque chose qui est caché, puis j'ai l'impression qu'ils m'envient un petit peu et ça me met mal à l'aise."

Cette distance se manifeste chez le patient qui peut à la fois éprouver une difficulté à bien comprendre le langage médical parfois hermétique et les recommandations du médecin, mais également ressentir une réticence à verbaliser ses peines et ses difficultés. Pour RSC4 : "J'aurais aimé trouvé une image qui illustrait la communication, le langage des personnes en situation de pauvreté versus nous. [...] Notre langage médical, je pense que ça peut être une grosse barrière. » Elle ajoute plus tard : «c'est clair que la majorité des gens [...] vivent beaucoup, beaucoup de difficultés. Puis sûrement qu'ils ne réussissent pas, les patients, à nous dire tout ce qu'ils vivent parce que c'est pas évident à reconnaître [...] et à le partager avec nous. »

D'un autre côté, une incompréhension de la part du professionnel de la santé face à son patient peut se manifester à la fois par un jugement hâtif sur le patient, qui ne prend pas en charge sa santé comme le médecin le souhaiterait, ou par des préjugés et présomptions qui ne tiennent pas compte de la situation réelle du patient. À ce sujet, RSC1 a verbalisé : «Je revoyais le pneumologue et le médecin gueuler contre les patients qui [ne respectent pas les traitements], 'Tu sais, [...] ils ne viennent même pas à notre centre d'éducation en asthme puis ils continuent à fumer! $\mathrm{RSC} 2$ a renchéri : "il y a toujours un petit préjugé en arrière de moi qui dit que la personne qui est en situation de pauvreté [...], j'ai l'impression que c'est des gens qui vont consommer de la drogue, qui boivent beaucoup d'alcool et qui fument. »

Dans le même ordre d'idées, plusieurs passages de la discussion font ressortir une différence de priorisation dans la vie des personnes en situation de pauvreté et des médecins, à la fois dans les choix matériels et dans les valeurs de base. D'après la discussion, cette barrière semble plus problématique au niveau de la prise en charge parce que le patient peut percevoir des besoins qui paraissent essentiels à ses yeux mais qui semblent futiles ou secondaires au médecin, et vice versa. RSC1 se questionnait : "Estce qu'il y a une certaine confusion entre les besoins primaires et secondaires [...] ils prennent Bell Express Vu [une antenne satellite payante] quand ils sont même pas capables d'avoir un chez-soi propre. [...] [lorsque je suis devant un itinérant] combien je donne? Est-ce qu'ils vont vraiment l'utiliser pour une bonne raison? On en voit beaucoup qui fument [...] est-ce qu'ils vont vraiment utiliser cet argent 


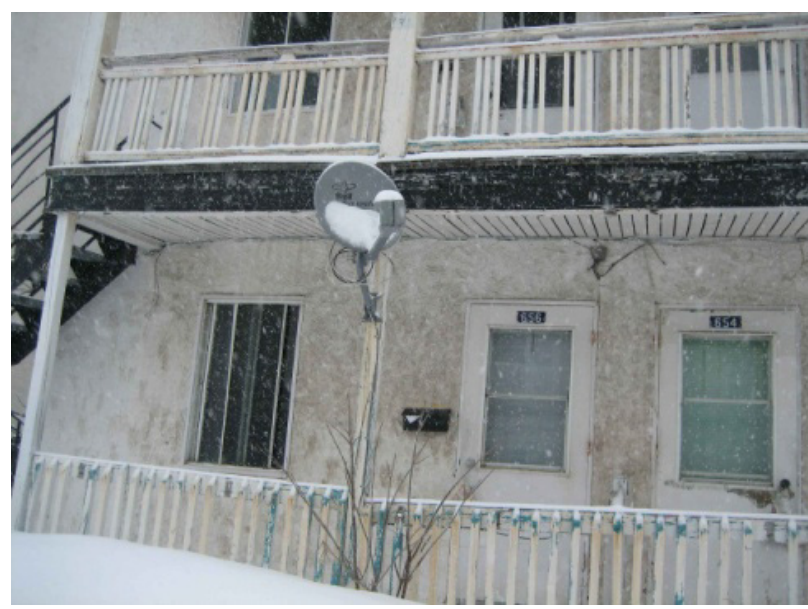

Photo 2. Priorisation des choix s: une antenne satellite payante.

pour ça et non pour des bonnes choses? » Elle a ajouté : "C'est une explosion de culture 'bling', tu sais, comme l'importance [pour les personnes en situation de pauvreté] de montrer des signes de richesse extérieure sur une pauvreté manifeste » (voir Photo 2).

Plusieurs émotions ressenties par les médecins et résidents face aux personnes en situation de pauvreté ont été identifiées lors de la rencontre, particulièrement la crainte des responsabilités rattachées à la prise en charge des personnes en situation de pauvreté et ce qu'elle implique. Pour RSC3, «l'obstacle dont ça m'a fait prendre conscience, c'est la responsabilité énorme. [...] Est-ce que comme médecin on n'a pas l'impression que quand on devient une personne significative dans un milieu où il y a un tel dénuement, on ne peut jamais partir sans abandonner? Quand on s'engage, c'est tellement exigeant, ça peut être tellement prenant, puis on sait qu'ils sont démunis... »Ceci a pour conséquence de créer un sentiment d'envahissement chez le résident qui peut être par la suite plus anxieux et affecté dans sa relation avec la personne en situation de pauvreté. Quant à RSC4, elle a mentionné : "Face à nos patients, ça devient un peu plus angoissant parce qu'on peut pas prendre toute la responsabilité de la solitude du patient sur nos épaules [...] Ça devient extrêmement lourd au quotidien. » RSC2 a également fait part de

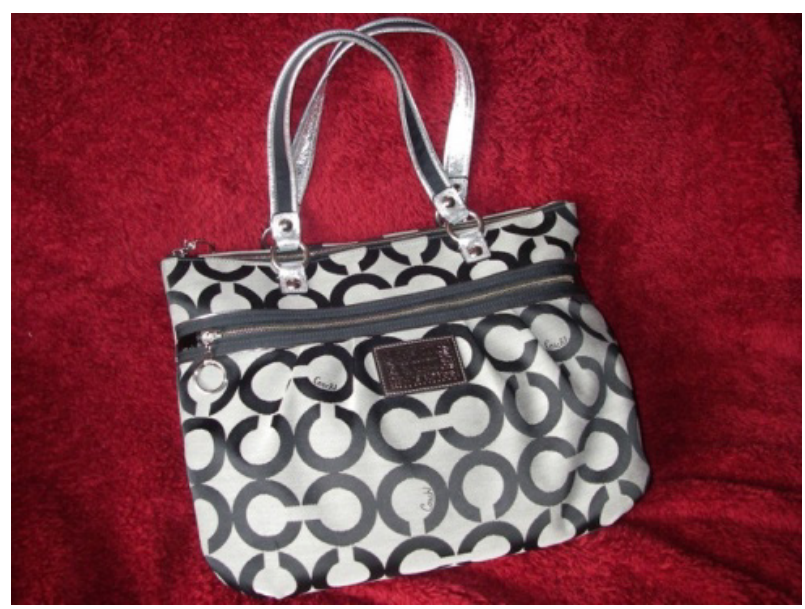

Photo 3. Distance sociale et consommation de biens « de luxe».

son sentiment d'impuissance en affirmant : «La pauvreté c'est toujours là, on n'y peut rien. "

De surcroit, un certain sentiment de honte de sa propre situation de part et d'autre a été dénoté à plusieurs reprises lors de la rencontre. Le patient et le médecin font tous deux l'objet d'une comparaison de statut social. Pour mieux comprendre l'autre et s'ajuster à sa différence, chaque individu tente à sa façon de diminuer la distance qui les sépare pour éviter le jugement de l'autre. RSC3 a avancé : «Lui [personne en situation de pauvreté], il met le vêtement le plus beau et il s'arrange pour être vraiment propre, la barbe rasée pour être bien sûr que [le médecin] va pas le juger parce qu'il a l'air pauvre». Et pour RSC1 : "Je [à titre de médecin] me sens un peu mal à l'aise d'avoir [...] des goûts luxueux puis d'être capable de m'acheter des choses qu'eux ne peuvent pas avoir. [...] Il y a l'envie, puis la jalousie, puis un petit peu la honte de ce qu'on a, mais il y a aussi la peur de se faire [voler]. [...] Est-ce que eux se posent autant de questions vis-à-vis nous? » (voir Photo 3).

Bref, la distance sociale qui existe entre les médecins et les personnes en situation de pauvreté se manifeste par différents obstacles en grande partie émotionnels, mais se traduit principalement par une difficulté à établir une alliance thérapeutique adéquate, provoquant ainsi une prise en charge qui ne 
tient pas assez compte des besoins et des attentes du patient.

Les solutions potentielles aux barrières entre le médecin en formation et la personne en situation de pauvreté

En plus des différentes barrières dans la relation patient-résident qui ont été présentées plus haut, le projet de photovoix a permis de faire ressortir des résultats émergents, capables de constituer des solutions à ces différentes barrières. Ces solutions ont été classifiées en différentes catégories : les solutions au niveau de l'éducation des résidents, au niveau personnel, au niveau relationnel, au niveau de la gestion d'aide et enfin au niveau social.

\section{Au niveau de la formation des résidents}

Il apparaît important de recevoir de la formation en ce qui a trait aux différentes ressources disponibles pour les personnes en situation de pauvreté, le manque de connaissance des ressources étant ressorti comme une barrière significative. À ce sujet, RSC3 a stipulé que "peut-être qu'un des cours sur la pauvreté pourrait être comme une appropriation du portrait de la pauvreté mais aussi des ressources qui existent autour. » Les RSC souhaiteraient également recevoir de la formation quant aux coûts et à la couverture des traitements qu'ils prescrivent. À la suite de la discussion sur la photo qui montre des médicaments que tous ne peuvent s'offrir par manque de ressources financières, RSC2 a commenté : "Je pense que c'est notre devoir de savoir à peu près ce qu'on prescrit, combien ça coûte, puis est-ce que c'est couvert ou non. »

\section{Au niveau personnel}

Finalement, le langage que les résidents doivent utiliser pour rejoindre efficacement leurs patients ressort comme un sujet de formation à retenir. Il est apparu que le fait d'avoir déjà été en contact étroit avec des personnes en situation de pauvreté, ou même de venir d'un tel milieu, est un des éléments principaux qui fait tomber les différentes barrières entre résident et patient. Cet élément a rejailli suite à la présentation de photos qui illustraient, selon les participantes, la relation privilégiée d'une résidente avec des personnes en situation de pauvreté.

RSC1 indique ainsi à RSC4 : "En fait, tes photos illustrent pas la barrière mais illustrent comment justement ces barrières-là sont déconstruites par le contact, comment ton contact avec la pauvreté à différents niveaux fait que ces barrières-là se déconstruisent et fait que tu connais c'est quoi la réalité de la pauvreté. »

\section{Au niveau relationnel}

Un bon contact relationnel est également une solution à privilégier avec les personnes en situation de pauvreté. Afin d'y parvenir, le résident doit d'abord être à l'écoute et ne pas juger son patient, comme l'a mentionné RSC3 en réponse aux différents préjugés que les résidents peuvent entretenir face aux personnes en situation de pauvreté : «Je pense qu'il y a des choses à faire. C'est juste qu'il faut apprendre à les écouter, puis à être ouvert». De même, RSC2 a ajouté plus tard dans la conversation qu' "il faut s'adapter à chaque patient » et "qu'il faut rester soi-même». Aussi, des actions plus concrètes peuvent permettre d'établir une meilleure relation résident-patient, tel que l'a énoncé RSC1: "Ça peut être des petites choses qui vont faire la différence, par exemple la manière qu'ils vont se vêtir, où est-ce que le patient va s'asseoir quand il rentre dans la salle de consultation, la communication, le langage, les mots qu'on va utiliser. Tout ça va faire que le patient [...] se sent [...] confortable de se confier. »

\section{Au niveau de la gestion d'aide}

La solution ayant trait à la gestion d'aide a été définie comme le fait de mieux connaître les ressources pour pouvoir y référer les patients efficacement. Cette solution passe donc par la formation, tel que mentionné plus haut, mais également par le fait que les résidents doivent reconnaître leurs limites et ne pas hésiter à référer aux ressources disponibles. Justement, lors d'une discussion portant sur le fait 
que les résidents se sentent parfois obligés de tout prendre sur leurs épaules, RSC 2 a affirmé : " [Il faut] être capable de dire, 'moi je peux pas aller plus loin pour t'aider de ce côté-là, mais regarde, il y a ça, il y a ça que tu pourrais faire, parce que [...] moi je ne peux pas aller plus loin'. » De même, RSC3 a rapporté une expérience positive qu'elle a vécue avec une personne en situation de pauvreté grâce à sa gestion d'aide : "J'ai vu [...] un immigrant depuis deux ans qui vit dans la pauvreté, sur l'aide sociale. [...] Je me sentais complètement démunie devant lui, je ne savais pas quoi lui dire. [...] Finalement on a trouvé des ateliers parent-enfant auxquels il pouvait participer, avec le CLSC [...] je trouvais que ça avait [...] aidé. Ça fait que je me suis sentie [...] un peu moins responsable. »

\section{Au niveau social}

La dernière solution mentionnée est de nature sociale voire sociétale. La première partie de cette solution reviendrait aux écoles de médecine qui devraient, selon RSC3, « sélectionner [...] des gens qui ont [...] une facilité à entrer en relation avec l'autre, une facilité à regarder leurs préjugés, à avoir de l'autocritique à propos de soi-même».

La deuxième partie de la solution incomberait plutôt au gouvernement qui, par le biais de la rémunération, ne favorise pas une prise en charge globale (et par le fait même, plus longue) des personnes en situation de pauvreté, comme il a été discuté plus tôt. Ainsi, il a été suggéré de payer les médecins à taux horaire plutôt qu'à l'acte afin d'encourager cette prise en charge.

\section{Discussion}

Ce projet de recherche selon la méthode photovoix a permis de faire ressortir diverses barrières dans la relation entre les médecins et des personnes en situation de pauvreté ainsi que des solutions potentielles pour diminuer celles-ci. D'abord, au niveau du contexte de la pratique médicale, il apparaît que le statut social propre au médecin, le manque de formation (principalement au niveau des ressources disponibles pour les personnes en situation de pauvreté), le manque de temps à consacrer à chaque patient et le manque d'incitatif salarial peuvent tous créer des difficultés importantes. Ensuite, il existe plusieurs barrières en lien avec le contexte socio-économique du patient, tels sa précarité économique, son milieu de vie parfois inadéquat, le coût des médicaments, son isolement géographique et son niveau de littératie. Enfin, la distance sociale entre le médecin et la personne en situation de pauvreté ne peut être oubliée ; le fait de venir de milieux différents, le caractère parfois hermétique du langage médical, les préjugés, les différences de priorisation et différentes émotions peuvent constituer de sérieuses entraves à la relation médecin-patient..

Cette étude est particulièrement intéressante du fait que la méthode photovoix a permis aux RSC de pousser leur réflexion plus loin qu'elles ne l'auraient fait sans passer par le processus photographique. Deux éléments ont permis ces résultats. D'une part, le photovoix, en posant des questions ouvertes lors de la présentation des photos en groupe, permet de saisir des événements dans leur contexte afin d'avoir une vision globale, avantage indéniable qu'apporte la recherche qualitative. D'autre part, les résidentes ont vu leur rôle habituel de personnes extérieures aux réalités des personnes avec qui elles travaillent être déstabilisé et cette déstabilisation a permis qu'elles passent de résidentes-chercheures à résidentessujets-chercheures, provoquant une réflexivité. Par conséquent, le photovoix a provoqué ce que Bolet ${ }^{[14]}$ appelle une "pédagogie de l'inconfort », manière d'être qui a eu pour conséquence d'augmenter la possibilité de connaissances des conditions réelles des personnes en situation de pauvreté par l'empathie des RSC.

Par ailleurs, le fait de prendre plusieurs photos et, par la suite, d'en sélectionner seulement cinq leur a permis de donner priorité aux idées les plus pertinentes. De la même manière, la question de recherche initiale étant ouverte et large, les RSC ont bénéficié d'une importante latitude dans la prise de photos et n'ont pas été limitées ou biaisées par une réponse attendue. Enfin, puisque toutes les résidentes venaient de milieux différents et avaient une 
grande diversité d'expériences avec les personnes en situation de pauvreté, cela leur a permis de faire ressortir des points de vue différents, mais complémentaires. De plus, la présence d'une sociologue et d'une anthropologue spécialiste du visuel lors de la discussion initiale a permis de diriger la rencontre sans s'égarer. La discussion a été enrichie par la présence d'un médecin qui amenait la perspective d'une clinicienne ayant une plus longue expérience clinique et par celle d'un psychologue qui teintait la discussion d'un point de vue différent.

Au niveau de l'analyse des données, une des forces de l'étude se situe dans le fait que la discussion a d'abord été analysée par les résidentes individuellement, puis en équipe de deux, et finalement rediscutée en groupe, ce qui a permis d'obtenir une validité intra- et inter-évaluateur. Une autre force de l'étude réside dans l'implantation des trois aspects primordiaux qui favorisent la nature participative de la recherche, selon Macaulay et al. ${ }^{[15]}$ dans le cadre d'une recherche-action. Ces trois éléments sont : 1) la collaboration entre les participants, les chercheurs et les intervenants du milieu où s'effectue cette recherche ; 2) une éducation mutuelle qui s'effectue entre toutes ces personnes lors de la définition de la question de recherche ainsi que lors de la collecte et de l'analyse de donnée et : 3 ) la possibilité « d'agir » pour transformer la communauté au sein de laquelle la recherche participative s'est déroulée.

Sur cette possibilité «d'agir», certaines des solutions proposées au cours de ce projet ont déjà été amenées par d'autres auteurs : le vécu du résident ou du médecin, l'approche relationnelle (aussi décrite comme approche centrée sur le patient ou compétence sociale dans la littérature) et les solutions sociales, notamment ${ }^{[2,6,7]}$. Cependant cette étude propose une piste de solution afin d'améliorer le soutien aux personnes en situation de pauvreté par la sollicitation de ressources communautaires adaptées lorsque leurs demandes excèdent les champs de compétences des médecins. Ainsi, à la lumière de cette étude, il apparaît primordial qu'un questionnaire relatif aux difficultés économiques et sociales du patient fasse partie intégrante de leur prise en charge.
Au niveau de la formation médicale, elle a également permis l'ajout d'un volet de formation explorant l'aspect de la pauvreté, à l'instar de ce que proposent d'autres chercheurs ${ }^{[3]}$.

Cette recherche a permis d'identifier des solutions, mais aussi d'amorcer le développement de mesures pédagogiques au sein du milieu académique. Les décideurs de la Faculté de médecine de l'Université de Sherbrooke à qui les RSC ont présenté leurs résultats de recherche ont entendu ces dernières : un cours dédié à la compréhension de la pauvreté a été ajouté au sein d'une unité de médecine de famille (UMF) et ce cours pourrait être dispensé plus largement aux autres UMF de la Faculté de médecine de l'Université de Sherbrooke. Par ailleurs, un parcours de formation qui vise à permettre aux résidents de visiter des organismes communautaires ayant pour clientèle des personnes en situation de pauvreté est en développement afin d'exposer les résidents à l'expertise du milieu communautaire et de développer chez eux le réflexe d'une médecine ancrée dans la communauté.

Bien que nous ayons utilisé la triangulation des données, des révisions systématiques par les pairs et l'écriture de journaux réflexifs pendant le processus de recherche, cette étude présente toutefois certaines limites. Les RSC ont eu peu de temps pour prendre leurs photographies, en plus d'être occupées à de nombreuses autres tâches cliniques et académiques, ce qui a pu limiter quelque peu leurs réflexions à ce sujet. De même, la retranscription du texte, bien qu'intégrale, était parfois incomplète, ce qui a pu entraîner l'omission de certaines informations importantes lors de l'analyse des données.

Finalement, il est à noter que l'étude présente uniquement les barrières du point de vue des résidents, et non sous l'angle des personnes en situation de pauvreté qui n'ont pas été impliquées dans le processus. Le but de l'étude était de mettre en lumière la perspective des médecins en formation ; il pourrait cependant être intéressant dans une étude future d'investiguer le point de vue des personnes en situation de pauvreté avec la méthode photovoix et de croiser ces perspectives avec celles des RSC. 


\section{Conclusion}

Ce projet de recherche a permis de faire ressortir plusieurs barrières entre les résidents et les personnes en situation de pauvreté. Il a également permis l'identification de solutions grâce au photovoix qui a généré une démarche réflexive permettant aux RSC de prendre connaissance, avec empathie, des conditions réelles de (sur)vie des personnes en situation de pauvreté. La première solution soulevée, et mise en place depuis, était l'élaboration d'un outil qui permettrait de référer les personnes en situation de pauvreté vers des ressources adaptées, et ce, dès le début de la prise en charge du médecin avec l'aide du questionnaire relatif aux difficulté économiques et sociales du patient. La deuxième solution proposée, et aussi implantée depuis, était le développement d'une formation à la diversité sociale et culturelle, qui semble souhaitable pour permettre aux médecins d'intégrer les déterminants sociaux à la pratique de la médecine familiale.

\section{Contributions}

Cet article a été inspiré en partie d'un rapport rédigé en 2011 dans le cadre d'un projet d'érudition scientifique par quatre résidentes en médecine de famille à l'Université de Sherbrooke: Annie-Claude Mailhot, Caroline Massicotte, Rébecca Millette et Julie Sirois-Leclerc. Les résidentes ont réalisé le recueil des données et effectué leurs analyses. Christine Loignon et Alexandrine Boudreault-Fournier ont supervisé la recherche et la rédaction du rapport des résidentes. Yanouchka Labrousse et Bruno Fortin ont participé au photovoix et ont commenté le travail des résidentes. Karoline Truchon a réalisé la mise en forme du rapport des résidentes en article.

\section{Approbation éthique}

Le protocole d'étude a été approuvé par le comité d'éthique du Centre hospitalier Universitaire de Sherbrooke (CHUS) et ce dernier n'a pas soulevé d'objection éthique.

\section{Déclaration d'intérêt}

Les auteurs déclarent qu'ils n'ont pas de conflit d'intérêt financier en lien avec l'objet de cet article.

\section{Références}

1. Raphael D. Poverty and policy in Canada. Implications for health and quality of life. Toronto: Canadian Scholars Publishers, 2007.

2. Woolhouse S, Belle Brown J, Lent B. Women marginalized by poverty and violence: How patient-physician relationships can help. Can Fam Physician 2004;50:1388-94.

3. Willems SJ, Swinnen W, De Maeseneer, JM. The GP's perception of poverty: a qualitative study. Fam Pract 2005;22:177-83.

4. Mercer SW, Watt GCM. The inverse care law: clinical primary care encounters in deprived and affluent areas of Scotland. Ann Fam Med 2007;5:503-10.

5. Wear D, Kuczewski MG. Medical Students' Perceptions of the Poor; What impact can medical education have? Acad Med 2008;83:639-45.

6. Loignon C, Haggerty J, Fortin M, Bedos C, Allen D, Barbeau D. Physicians' social competence in the provision of care to persons living in poverty: research protocol. BMC Health Serv Res 2010a; 10(79).

7. Loignon C, Haggerty J, Fortin M, Bedos C, Barbeau $\mathrm{D}$, Allen D. What makes primary care effective for people in poverty living with multiple chronic conditions?: study protocol. BMC Health Serv Res 2010b;10(320).

8. Loignon C, Haggerty J, Fortin M, Bedos C, Allen D, Barbeau D. Physicians' social competence in the provision of care to persons living in poverty: research protocol. BMC Health Serv Res 2010a.

9. Loignon C, Boudreault-Fournier A. La flexibilité des médecins offrant des soins de première ligne aux personnes en situation de pauvreté : la compétence sociale revisitée. Health Care Policy 2013;9(Special issue):59-70.

10. Loignon C, Boudreault-Fournier A. From Paternalism to Benevolent Coaching. Can Fam Physician 2012; 58:1194-95. 
11. Catalani C, Minkler M. Photovoice: A Review of the Literature in Health and Public Health. Health Educ Behav 2009;37:424-51.

12. López EDS, Eng E, Robinson N, Wang CC. Photovoice as a Community-Based Participatory Research Method. Methods in Community-Based Participatory Research for Health 2005;324-45.

13. Hsieh H, Shannon SE. Three Approaches to Qualitative Content Analysis. Qual Health Res 2005;15: 1277-88.

14. Bolet cité dans Wear D, Kuczewski MG. Medical Students' Perceptions of the Poor: What impact can medical education have? Acad Med 2008;83: 639-45.

15. Macaulay AC, Commanda LA, Freeman WL, Gibson N, McCabe ML et al. Participatory research maximises community and lay involvement. BMJ 1999; 319:774-78.

Correspondance et offprints: Christine Loignon, 150 Place Charles Lemoyne Bureau 200, Longueuil, Québec, Canada J4K 0A8.

Mailto: christine.loignon@usherbrooke.ca 